\title{
Structural insight on the Hsp90-binding modes of Naphthoquinone derivatives. Molecular Modeling study for antitumoral drug design.
}

\author{
Claudio Godoy-Castillo ${ }^{1,2}$, Jorge F. Gonzalez ${ }^{1}$ and Jorge Soto-Delgado ${ }^{\mathbf{1}}$ \\ 1. Departamento de Ciencias Químicas, Facultad de Ciencias Exactas, Universidad Andrés \\ Bello, Quillota 980,Viña del Mar, Chile.E-mail: jorge.soto@unab.cl \\ 2: Escuela de Química y Farmacia, Facultas de Medicina, Universidad Andrés Bello, \\ Quillota 980, Viña del Mar, Chile.
}

\section{ABSTRACT}

Heat shock proteins (HSPs) constitute a large family of proteins involved in protein folding and maturation whose expression is induced by heat shock or other stressors. HSPs play important roles in carcinogenesis via the regulation of angiogenesis, cell proliferation, migration, invasion, and metastasis. One of the most attractive targets for novel antitumor agents during recent years is the Heat shock proteins 90 (Hsp90), which is an essential molecular chaperone and it is responsible for the folding and maturation of nascent proteins. ${ }^{1}$ In this context, inhibition of Hsp90 has potential to disrupt multiple oncogenic pathways by an indirect attack on critical proteins and has become an attractive target for cancer therapy. ${ }^{2}$

This work aims to develop of new molecules based on quinone scaffold due that quinones derivatives have demonstrated potent anti-proliferative activity against estrogen-dependent cancers cell, as well as, induce the degradation of oncogenic Hsp90 client proteins. The set of 35 molecules reported by Blagg et $\mathrm{al}^{3}{ }^{3}$ were studied based in a hybrid strategy including Molecular Docking, Molecular Dynamics and 3D-QSAR analysis to identify the most relevant ligand-receptor interactions. ${ }^{4}$ The Correlation between the free energy of binding and binding modes for nafthoquinones derivatives are discussed. These results provide a new insight into protein-ligand interactions into the Hsp90, in addition, the analysis of the contour maps derived for 3D-QSAR provide helpful way about the rational modification of molecules in order to design more potent Hsp90 inhibitors.

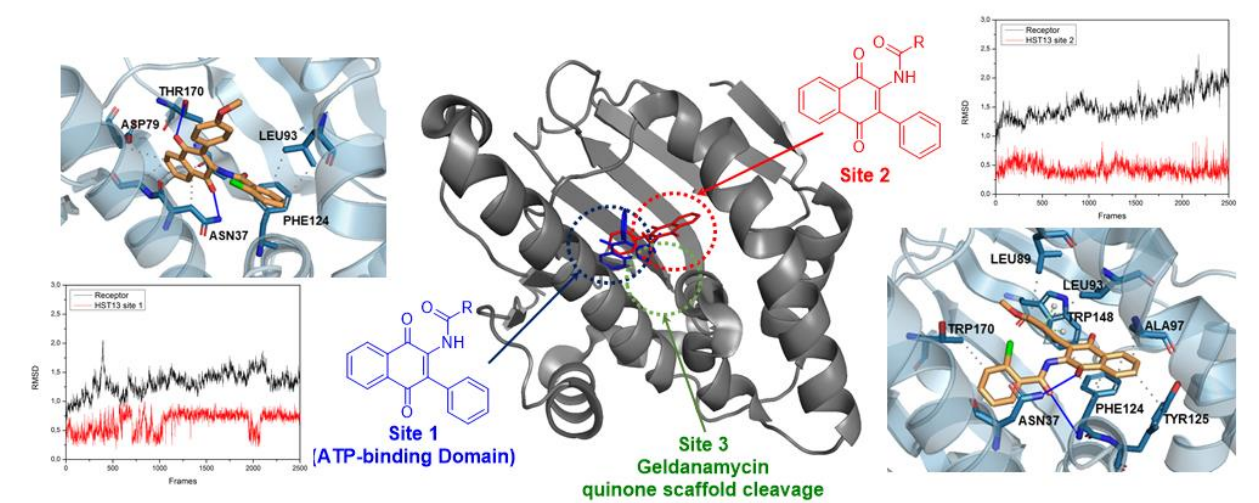

Acknowledgements: Work supported by project FONDECYT Iniciación Grant 11150988 and Universidad Andrés Bello (UNAB) research grant DI-1292-16/R.

\section{References.}

1. Wu, J.; Liu, T.; Rios, Z.; Mei, Q.; Lin, X.; Cao, S. Trends Pharmacol. Sci. 2017, 38, 226.

2. (a)Taldone, T.; Ochiana, S.O.; Patel, P.D.; Chiosis, G. Trends Pharmacol. Sci. 2014, 35, 592 (b) Jhaveri, K.; Taldone,

T.; Modi, S.; Chiosis, G. Biochim. Biophy. Acta, 2012, 1823, 742.

3. Hadden, M.K.; Hill, S.A.; Davenport, J.; Matts, R.L.; Blagg, B.S.J. Bioorg. Med. Chem. 2009, 17, 634.

4. Lopez-Lira, C., Alzate-Morales, J.H., Paulino, M., Mella-Raipán, J., Salas, C.O., Tapia, R.A., Soto-Delgado, J. Chem. Biol. Drug. Des. 2017 DOI:10.1111/cbdd.13051 